\title{
Zwischen den Ordnungen
}

Liebe Leserinnen, liebe Leser,

das menschliche Leben ist geordnet und geregelt. Die Ordnung beruht auf gesellschaftlichen Übereinkünften und kostet dem Individuum Freiheit. Sie sieht vor, dass es sich den Regeln unterwirft und dass inm dafür beim Überleben geholfen wird. Sie ist parzelliert und geschichtet: Familie, Arbeitsplatz-, Verein etc.; orthogonal dazu Anstandsregeln, Moral, Rechtsordnung etc. Die jeweiligen Regeln halten sich an die ethische Grundhaltung der Gesellschaft; so sind sie miteinander verträglich. Dafür, dass sie das freiheitslustige Individuum einhält, sorgt jeweils eine spezifische Autorität - Familie, Arbeitgeber, Polizei etc. Wer gegen den Druck dieser Autoritäten seine Freiheit sucht, wird dabei beharrlich behindert. Besteht er dennoch darauf, droht ihm in der Konsequenz die Not des Asozialen. Mit Renitenz mag er jedoch dazu beitragen, dass sich die Regeln mit der Zeit ändern. Soweit die Erfahrung. Zu all dem entwickelt die Gesellschaft eine passende Staatslehre, z.B. die von der Demokratie. Diese erlässt Gesetze, die sich an überlieferte Wertvorstellungen und an die vom Staatsvolk renitent vorgebrachten Veränderungswünsche halten. Der Staat erzwingt die Einhaltung der Gesetze. Alles in allem: eine Ordnung, welche die Menschenrechte gut bedient und deshalb als gerecht erscheint.

Anders verhält es sich im internationalen Bereich. Dort stehen die Egoismen der Staaten gegeneinander und wird im Ernstfall Krieg geführt. Das wird zwar als misslich empfunden; es fehlt aber eine legitime Gewalt, die Übeltaten verhindern kann. Zwar werden unter den Beteiligten Abkommen geschlossen, aber deren Signatare fühlen sich allein den Interessen ihres Staatsvolks verpflichtet. Um sicher zu gehen, bewaffnet sich jeder Staat und/oder sucht Verbündete. Das führt zu Zweckbündnissen und zu einem dynamischen Gleichgewicht; zu einer Ordnung, die auf Macht und Misstrauen beruht. Sie entspricht der Default-Position, die von der Evolution durchlaufen wurde und für den Fall bereitgehalten wird, dass die höhere Ordnung zusammenbricht. In dieser archaisch-primitiven Ordnung sind die Menschenrechte sekundär; diese Ordnung erscheint als ungerecht.

Zur Kompensation des Vertrauensdefizits unterhalten die Staaten neben ihrem Militär auch nationale Nachrichtendienste, die alles beobachten sollen, was das nationale Wohl betreffen könnte. Der Konkurrenzsituation folgend agieren die Dienste grundsätzlich gegeneinander und verfolgen national-egoistische Ziele. Im Falle von Bündnissen kooperieren sie nur so weit, wie es die Vereinbarungen vorsehen. Sie suchen im Zweifelsfalle überall Kenntnisse über alles zu erlangen, was das Wohl des Staates betreffen könnte und sparen dabei das eigene Staatsvolk nicht aus. Notwendigerweise tun sie das unter den Umständen der Ordnung zweiter Art und naturgemäß im Geheimen. Damit dienen sie ihren Regierungen dazu, der internationalen Ordnung ohne unnötiges Aufheben zu genügen und Schaden vom eigenen Staatsvolk abzuwenden.

Eine Regierung muss also zwei miteinander unverträgliche Ordnungen bedienen. Sie muss einerseits dem Staatsvolk gegenüber die demokratischen Werte sichern und andererseits - auch unter Hintanstellung solcher Werte - im internationalen Hauen und Stechen bestehen. Sie muss, um die Geheimhaltung vor den Konkurrenten zu sichern, darauf verzichten, von ihrem Nachrichtendienst alles unmittelbar zu erfahren, und riskiert damit, dass von ihm demokratische Werte verdeckt verletzt werden. Mit seinem Wissen ist er ihr zugleich unersetzlich; deshalb hält sie von ihm Schaden fern. Wenn dennoch Verdecktes bekannt wird, gibt es öffentlichen Aufklärungsbedarf. Dieser führt in der Regel zu einer Präzisierung demokratischer Werte und zu Regelungsbedarf. Mit neuen Regeln wird die Grenze zur internationalen Ordnung befestigt und damit das Ausmaß der unbewältigten Spannungen verdeutlicht. Die Grenze zwischen den Ordnungen bleibt ungeordnet.

Diese Grenze, liebe Leserinnen und Leser, trägt mit immer neuen Impulsen zur Verbesserung der demokratischen Ordnung bei und bietet mit der jenseits angebotenen evolutionären Default-Position auch die Garantie dafür, dass die Entwicklung weiter geht.

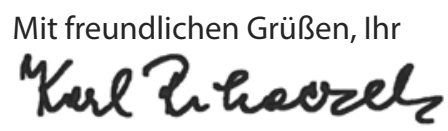

